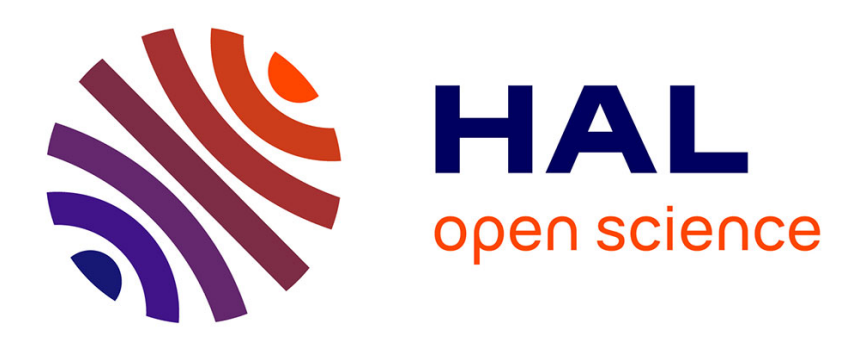

\title{
Quelques concordances entre le Liet von Troye et le manuscrit A1 du Roman de Troie
}

Marie-Sophie Masse

\section{To cite this version:}

Marie-Sophie Masse. Quelques concordances entre le Liet von Troye et le manuscrit A1 du Roman de Troie. Romania (Paris), 2003, 121, pp.218 - 236. 10.3406/roma.2003.1291 . hal-03372830

\section{HAL Id: hal-03372830 \\ https://hal-u-picardie.archives-ouvertes.fr/hal-03372830}

Submitted on 11 Oct 2021

HAL is a multi-disciplinary open access archive for the deposit and dissemination of scientific research documents, whether they are published or not. The documents may come from teaching and research institutions in France or abroad, or from public or private research centers.
L'archive ouverte pluridisciplinaire HAL, est destinée au dépôt et à la diffusion de documents scientifiques de niveau recherche, publiés ou non, émanant des établissements d'enseignement et de recherche français ou étrangers, des laboratoires publics ou privés. 


\section{Quelques concordances entre le Liet von Troye et le manuscrit A1} du Roman de Troie

Marie-Sophie Masse

\section{Citer ce document / Cite this document :}

Masse Marie-Sophie. Quelques concordances entre le Liet von Troye et le manuscrit A1 du Roman de Troie. In: Romania, tome $121 \mathrm{n}^{\circ} 481-482,2003$. pp. 218-236;

doi : 10.3406/roma.2003.1291

http://www.persee.fr/doc/roma_0035-8029_2003_num_121_481_1291

Document généré le 16/11/2016 


\section{MÉLANGES}

\section{QUELQUES CONCORDANCES ENTRE LE LIET VON TROYE ET LE MANUSCRIT A1 DU ROMAN DE TROIE*}

Rédigé par Herbort von Fritzlar à la cour de Thuringe entre 1190 et $1217^{1}$, le Liet von Troye ${ }^{2}$ constitue la première translation en langue allemande du Roman de Troie de Benoît de Sainte-Maure ${ }^{3}$. Ce récit d'Antiquité s'inscrit dans la tradition d'《 adaptation créative ${ }^{4}$ " de romans en langue française dans l'aire germanophone, née au milieu $\mathrm{du}$ XII $^{\mathrm{e}}$ siècle et restée vivace jusqu'à la fin du XIII ${ }^{\mathrm{e}}$ siècle. Procédé de récriture, l'adaptation peut être étudiée avec fruit en regard de sa source. Mais dès que l'on souhaite aborder cette comparaison sur une base rigoureuse, l'on se trouve confronté à la «mouvance» de tout

*Cet article doit beaucoup à l'aide précieuse de $\mathbf{M}^{\mathrm{me}}$ Françoise Vielliard.

1. Ce sont en effet les dates du règne du landgrave Hermann $I^{\text {er }}$ de Thuringe, mécène de Herbort.

2. Dans l'attente de l'édition préparée par J. Bumke, la seule édition du texte de Herbort reste celle de K. Frommann: Herbort's von Fritslâr liet von Troye, Amsterdam, 1966 [reproduction de l'édition de 1837]. Cette édition sera désignée dans la suite de l'analyse sous le titre Liet von Troye.

3. L'édition de référence est celle de $\mathbf{L}$. Constans (désormais désignée sous le titre Le Roman de Troie) : Le Roman de Troie de Benoît de Sainte-Maure, Paris, 1904-1912 [SATF], 6 volumes.

4. Le terme a été forgé par $D$. Buschinger sur le modèle du terme allemand « Nachdichtung » (D. Buschinger, «Die Kontroverse um die 'adaptation courtoise' ", dans Kontroversen, alte und neue. Akten des VII. Internationalen Germanisten-Kongresses Göttingen 1986. Bd. 9 : Deutsche Literatur in der Weltliteratur — Kulturnation statt politischer Nation, Tübingen, 1986, p. 133-134). 
texte transmis dans plusieurs versions manuscrites. La recherche du manuscrit-source s'avère particulièrement délicate dans le cas du Liet von Troye, qui ne présente pas seulement la mise en œuvre nouvelle propre à toute adaptation : de plus, à rebours des autres adaptateurs allemands, qui amplifient leur source, Herbort l'abrège presque de moitié ${ }^{5}$. L'abbreviatio pratiquée par l'auteur accroît naturellement la difficulté de l'analyse comparative qui peut être menée entre le Liet von Troye, transmis par un unique manuscrit complet ${ }^{6}$, et Le Roman de Troie, transmis par trente manuscrits complets et vingt-huit fragments ${ }^{7}$.

La seule étude consacrée à la question du manuscrit-source du Liet von Troye est due à Hermann Menhardt : dans un article paru en $1928^{8}$, le critique affirme qu'il s'agit du manuscrit $A 2$ (Paris, Arsenal $3342^{\circ}$ ), l'un des plus anciens manuscrits du Roman de Troie ${ }^{10}$.

5. Le texte de Herbort comporte 18458 vers dans l'édition de K. Frommann, celui de Benoît 30316 vers dans l'édition de L. Constans. Le Liet von Troye constitue le seul cas d'abréviation patente dans le cadre de la littérature courtoise, comme l'indique J. Bumke (J. Bumke, Die romanisch-deutschen Literaturbeziehungen im Mittelalter. Ein Überblick, Heidelberg, 1967, p. 29).

6. Il s'agit du manuscrit $H$, Heidelberger Universitätsbibliothek Cod. pal. germ. 368. Sont connus par ailleurs trois fragments du texte: $B$ Berliner Fragmente Mgf 902. Bl. $1+2$ (v. 13017-13290 et 14379-14641), édité par J. Bumke, « Untersuchungen zu den Epenhandschriften des 13. Jahrhunderts. Die Berliner Herbortfragmente », dans Zeitschrift für deutsches Altertum und deutsche Literatur, t. 119 (1990), p. 404-434 ; S Skokloster, Schloßbibliothek., Cod. PB munk 4 (v. 7735-8512), édité par H. Psilander, « Ett fragment af den tyska Trojasagan i det Wrangelska biblioteket på Skokloster », Uppsala, Akademiska Boktryckeriet, 1917 [Uppsala Universitets Arsskrift 1917, Progr. 7] ; K Krumau, Zweigstelle des Staatlichen Regionalarchivs Wittingau (v. 799-915, 935-959, 1256, 1365-1401, 1532-1568), édité par V. Bok / H. Boková / K. Gärtner, « Neue Herbortfragmente aus Krumau », dans Beiträge zur Geschichte der deutschen Sprache und Literatur, t. 118 (1996), p. 333-357.

7. Nous nous fondons sur la liste établie par M.-R. Jung dans son ouvrage $L a$ légende de Troie en France au Moyen Âge. Analyse des versions françaises et bibliographie raisonnée des manuscrits, Basel / Tübingen, 1996, p. 22-23 [Romanica Helvetica, 114].

8. H. Menhardt, "Herbortstudien ", dans Zeitschrift für deutsches Altertum und deutsche Literatur, t. 65 (1928), p. 225-254.

9. Sur le manuscrit $A 2$, voir l'ouvrage de M.-R. Jung, op. cit., p. 139-147.

10. $A 2$ date peut-être du $\mathrm{XII}^{\mathrm{e}}$ siècle. 
Toutefois Hermann Menhardt n'avait pas accès au manuscrit luimême mais travaillait sur l'édition procurée au début du siècle par Léopold Constans ${ }^{11}$. Ces conditions de travail sont à l'origine de deux erreurs dans les arguments qu'il développe.

D'une part, en effet, le critique fait référence à ce qu'il nomme un "passage célèbre" du Liet von Troye ${ }^{12}$ : en présentant la famille de Priam à la veille de la reconstruction de Troie détruite par les Argonautes, l'adaptateur nomme la fille aînée de Priam «Creusa », et non "Andromacha » selon la leçon donnée par Le Roman de Troie. On en a déduit, rappelle Hermann Menhardt, que Herbort avait eu recours à l'Énéide afin de corriger l'erreur commise par Benoît à la suite de Darès. Le critique s'inscrit en faux contre cette hypothèse car, affirme$\mathrm{t}$-il, il existe un manuscrit du Roman de Troie qui comporte déjà «Creusa »: le manuscrit $A 2$. Or l'argument est fondé sur une erreur qu'il reprend à Léopold Constans ${ }^{13}$ : la vérification effectuée sur le manuscrit $A 2$ montre que celui-ci comporte non "Creusa » mais «Andromacha 14 ».

Hermann Menhardt consacre d'autre part un long développement à la présence de trois personnages portant le nom d'《 Ayax » dans le Liet von Troye. Il explique en effet ce phénomène par une particularité de $A 2{ }^{15}$ : afin de corriger une erreur de Benoît, qui réintroduit Ajax fils de Télamon dans la vingt-et-unième bataille, alors qu'il est tué par Pâris lors de la bataille précédente, le copiste de $A 2$ aurait introduit trois Ajax dans le résumé du récit situé au début du Roman de Troie. Selon Hermann Menhardt, qui prend appui sur les variantes consignées par Léopold Constans ${ }^{16}, A 2$ substituerait le nom d'Ajax à celui de Ménélas dans ces vers du résumé :

11. Voir la note 3.

12. H. Menhardt, art. cit., p. 238.

13. Voir les commentaires de L. Constans au sujet du manuscrit $A 2$ : « Ce scribe était d'ailleurs instruit et ne manquait pas l'occasion de donner la preuve de son érudition. [...] Au v. 2950, au lieu de 'Andromacha', l'aînée des filles de Priam et d'Hécube, il met 'Creüsa' (rectifiant ainsi Darès), et il ajoute : 'qui ert mariee', ce qui est exact » (Le Roman de Troie, t. VI, p. 26).

14. $A 2$ comporte au f. $15 \mathrm{~d}:$ : des .III. filles ot non lains nee / andromacha moult fu senee ».

15. H. Menhardt, art. cit., p. 234-238.

16. Le Roman de Troie, t. I, p. 458 , variante de la page $11,1.13$ et 1.14 . 
Qu'Agamennon e Menelaus,

e Telamon e Aïaus,

Palamedés e Ulixés,

li dus d'Athene e Achillés,

e cent riche rei e preisié

vindrent sor Troïens irié.

(éd. Constans, v. 189-194)

Or après vérification, il s'avère que le manuscrit $A 2$ comporte "Menelaus 17 » et non "Aiaus", et mentionne donc uniquement, comme les autres manuscrits, deux Ajax : Ajax fils d'Oỉlée et Ajax fils de Télamon.

Ces constats invitent à reconsidérer la question du manuscrit ou de la famille de manuscrits qui a pu constituer la source du Liet von Troye. Car la voie ouverte par Hermann Menhardt n'a pas été suivie plus avant : Hans Fromm, plus de cinquante ans plus tard, déplore que son hypothèse ne soit toujours ni confirmée ni infirmée ${ }^{18}$. Or dans ce domaine, l'on dispose désormais de cet outil précieux qu'est le livre consacré par Marc-René Jung à la tradition manuscrite du Roman de Troie ${ }^{19}$. La lecture croisée de cet ouvrage et du Liet von Troye laisse à penser que le texte de Herbort est plus proche des manuscrits de la deuxième famille, et notamment qu'il présente un certain nombre de leçons communes avec le manuscrit $A 1$ (Paris, Arsenal 3340). C'est cette hypothèse de travail que nous nous proposons d'explorer.

Fait remarquable si l'on songe au «passage célèbre » cité par Hermann Menhardt, Marc-René Jung signale que deux manuscrits du Roman de Troie substituent le nom de Créüse à celui d'Andromaque

17. $A 2$ comporte au f. $1 \mathrm{c}:$ « quagamenon e menelaus / e thelamon e rois aiaus / protheselaus e ulixes $»$.

18. H. Fromm, "Herbort von Fritslar. Ein Plädoyer », dans Beiträge zur Geschichte der deutschen Sprache und Literatur, t. 115 (1993), p. 244-278, en particulier p. 247. L'auteur précise (note 7 , p. 247) que H. Franz a remis en cause l'hypothèse de $\mathbf{H}$. Menhardt à partir de l'étude des noms propres. $\mathrm{H}$. Franz réfute de fait l'idée d'une source romane unique fournie par $A 2$, sans toutefois établir d'autre hypothèse, car tel n'est pas son propos (H. Franz, Das Bild Griechenlands und Italiens in den mittelhochdeutschen epischen Erzählungen vor 1250, Berlin, 1970 [Philologische Studien und Quellen, 52], en particulier p. 387).

19. Voir la note 7. 
dans la présentation des filles de Priam ${ }^{20}$. Il s'agit de $A l$ et de $L 2$ (Londres, BL Add. 30863), deux manuscrits de la deuxième famille, section $z$ d'après la classification établie par Léopold Constans ${ }^{21}$ :

Des trois filles ot non l'einnee Creüsa, qui ert mariee: molt fu bele, molt fu cortoise, moll ama anor et prooise 22 .

$$
(A 1, \text { f. 18b) }
$$

Des treis filles ot non l'ainz nee Andromacha : mout fu senee, mout fu bele, mout fu corteise, mout ama honor e proëise.

(éd. Constans, v. 2949-2952)

Mis en regard, le texte du Liet von Troye, qui nomme Créüse et signale qu'elle est mariée, se situe nettement du côté des manuscrits $A l$ et L2 :

$$
\begin{aligned}
& \text { Priamus wip hiz Ecuba, } \\
& \text { sin grozzer tochter Creusa, } \\
& \text { Polixena die andra, } \\
& \text { die dritte hiz Cassandra. } \\
& \text { Creusa hette einen man. } \\
& \text { Cassandra liz sich wizigen an } 23 \text {. } \\
& \text { (v. 1689-1694) }
\end{aligned}
$$

L'argument développé par Hermann Menhardt sur ce point s'avère donc jouer en faveur de la branche représentée par $A 1$ et $L 2{ }^{24}$.

Marc-René Jung indique que les manuscrits $A 1$ et $L 2$ ont en commun une autre leçon qui leur est propre ${ }^{25}$ : Galatée, le cheval d'Hector présenté lors de la seconde bataille, n'est pas le don de la fée Orva, leçon des autres manuscrits, mais celui de Penthésilée :

20. M.-R. Jung, op. cit., p. 42 et p. 102. 26.

21. La classification est reprise et complétée par M.-R. Jung, op. cit., p. 24-

22. Toutes les citations de $A 1$ sont des transcriptions faites à partir du manuscrit, que nous avons pu consulter à la Bibliothèque de l'Arsenal. Nous remercions à cette occasion les conservateurs qui nous ont permis d'avoir accès au manuscrit.

23. Liet von Troye, p. 20 ( « La femme de Priam se nommait Hécube, sa fille aînée Créüse, l'autre Polyxène, et la troisième se nommait Cassandre. Créüse était mariée. Cassandre se fit prophète ").

24. Il y a donc peut-être eu confusion de la part de L. Constans entre les manuscrits $A 1$ et $A 2$.

25. M.-R. Jung, op. cit., p. 17 (note 3), p. 47 et p. 102. 
Hector monta sor Galatee, que li tramist Panthesilee, qui molt l'ama et molt l'ot chier : por ce li dona cel destrier.

$(A 1$, f. 50a)
Hector monta sor Galatee, que li tramist Orva la fee, que mout l'ama e mout l'ot chier mais ne la voust o sei couchier.

(éd. Constans, v. 8023-8026)

Sur cc point encore, le texte de Herbort concorde avec $A l$ et L2:

Ector uf ein ros saz

harte gut. Man saget, daz

ez hieze Galathea.

Ez sante im Pentesilea,

ein hubische juncfrouwe ${ }^{26}$.

(v. 4791-4795)

Ainsi le manuscrit du Liet von Troye possède-t-il les deux leçons propres aux manuscrits $A I$ et $L 2$ du Roman de Troie. L'on pourrait, bien sûr, objecter que Herbort, nourri de culture antique, a pu corriger de lui-même le nom d'Andromaque en celui de Créüse. L'on pourrait avancer aussi qu'il a pu substituer Penthésilée à la fée Orva en regard de l'entrevue d'Achille et d'Hector rapportée au terme de la huitième bataille, car le texte allemand indique alors, à la suite du Roman de Troie, que le vêtement d'Hector est un cadeau fait par la reine des Amazones ${ }^{27}$. Mais d'autres indices montrent une parenté entre le Liet von Troye et le manuscrit $A 1^{28}$ et portent à croire que ces corrections proviennent du manuscrit-source de Herbort.

26. Liet von Troye, p. 55 (« Hector montait un excellent cheval. On dit qu'il s'appelait Galatée. C'est Penthésilée, une demoiselle courtoise, qui le lui avait fait parvenir $")$.

27. Liet von Troye, v. 8194-8197, p. 94. Dans Le Roman de Troie, le détail ne se trouve que dans la version longue du passage, que comporte notamment le manuscrit $K$ édité par A. Joly (A. Joly, Benoît de Sainte-More et Le Roman de Troie ou les métamorphoses d' Homère et de l'épopée gréco-latine au Moyen Âge, Paris, t. II, 1871, v. 12987-13234, p. 184-187; le passage est retranscrit par L. Constans, Le Roman de Troie, t. IV, p. 399-409).

28. La comparaison entre les particularités de $L 2$ notifiées par M.-R. Jung (op. cit., p. 100-110) et le Liet von Troye ne nous semble pas concluante. Les nombreuses omissions qui caractérisent $L 2$, en particulier celle de la longue description de l'Orient, ne se retrouvent pas dans le texte de Herbort. 
Outre les leçons particulières et communes aux manuscrits $A 1$ et $L 2$, Marc-René Jung relève quatre leçons propres à $A 1^{29}$. L'une d'elles ne paraît pas probante dans le cadre de la comparaison avec le Liet von Troye : elle concerne l'excuse formulée par Benoît auprès de la « riche dame de riche rei », sans doute Aliénor d'Aquitaine, après la digression que l'auteur consacre à l'inconstance féminine ${ }^{30}$; or le texte de Herbort ne comporte pas cette excuse. Une autre lcçon sc situe dans l'entrevue d'Hector et d'Achille, pour laquelle la tradition manuscrite du Roman de Troie offre deux versions, l'une brève, l'autre longue. $A I$ mêle les deux rédactions, commençant par la version longue (introduction narrative et répliques d'Achille et d'Hector au discours direct) pour continuer par la rédaction brève (réplique d'Achille au discours direct), avant de donner la fin narrative longue (opposition des chefs grecs et troyens au duel voulu par les protagonistes ${ }^{31}$ ). S'il est possible de vérifier que le passage, chez Herbort, correspond au même schéma d'ensemble ${ }^{32}$, il est ensuite difficile de poursuivre l'analyse plus avant tant l'adaptateur allemand abrège sa source. En revanche, les deux autres leçons signalées par Marc-René Jung, qui prennent place dans l'épisode de la vengeance de Nauplius et dans le dénombrement des alliés de Priam, recèlent dans le détail des indices supplémentaires en faveur de $A 1$.

L'épisode de la vengeance de Nauplius se situe dans le récit des " retours » qui clôt le roman. Apprenant que son fils Palamède a été tué par Ulysse, Nauplius fait allumer un feu sur Eubée afin d'attirer les Grecs. Lorsqu'ils s'approchent de la côte, Nauplius les accueille par

29. M.-R. Jung, op. cit., p. 137-139.

30. Le Roman de Troie, t. II, v. 13457-13470, p. 302-303. M.-R. Jung remarque que dans $A I$, l'excuse ne s'adresse pas à une riche dame, mais à Dieu, et se termine par une sorte d'invocation à la Vierge (op. cit., p. 138).

31. On pourra se reporter à l'analyse que consacre M.-R. Jung à cet épisode et aux différentes versions (op. cit., p. 30-32 et, sur $A I$, p. 138).

32. Le texte de Herbort se présente de la façon suivante : 1. Introduction narrative (v. 8173-8206) : version longue. - - 2. Discours d'Achille et d'Hector (v. 8207-8274) : version longue. - 3. Réplique d'Achille (v. 8275-8284) : version courte. - 4. Fin narrative (v. 8285-8306): version longue.

Dans le détail, Herbort supprime de nombreux éléments, par exemple l'évocation des chevaliers qui accompagnent les deux protagonistes ou les accusations de pédérastie formulées par Hector, et il abrège l'évocation de la réaction du public à la fin de la scène. 
une pluie de rochers. Or cette vengeance est suscitée par un mensonge, puisque, selon les données du texte, Palamède a été tué par Pâris lors de la douzième bataille. Deux tiers des manuscrits du Roman de Troie le suggèrent en employant le verbe "engignier ${ }^{33}$ », mais $A 1$, dans un développement, précise en outre expressément que le récit fait à Nauplius est faux :

Si li avoit an reconté
autrement qu'il n'avoit esté :
dit li estoit que Ulixés
et ses compeinz Dyomedés
orent tant porchacié et quis
que antr'aus l'avoient ocis ${ }^{34}$.

$(A 1, \mathrm{f} .172 \mathrm{c})$

Le texte du Liet von Troye précise également dans le même passage que le bruit répandu est un mensonge :

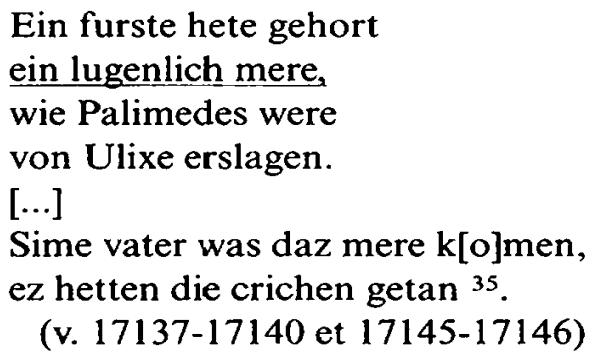

Par ailleurs, dans sa description du manuscrit $A I$, Marc-René Jung signale que celui-ci présente certaines omissions particulières, peu nombreuses mais fâcheuses ${ }^{36}$. Il en donne un exemple avec le dénombrement des alliés de Priam situé avant le débarquement des Grecs et la première bataille ${ }^{37}$. Le manuscrit $A l$ passe sous silence douze vers qui

33. Voir M.-R. Jung, op. cit., p. 72 et p. 138.

34. L'ajout développe les vers $27685-27686$ du texte édité par L. Constans (Le Roman de Troie, t. IV, p. 247). L. Constans, déjà, signale dans ses « variantes complémentaires " que $A I$ développe ce passage (ibid., t. IV, p. 436).

35. Liet von Troye, p. 196 ( Un prince avait entendu des propos mensongers selon lesquels Palamède aurait été tué par Ulysse. [...] Ces propos selon lesquels cela aurait été le fait des Grecs étaient parvenus à son père ").

36. M.-R. Jung, op. cit., p. 139.

37. Le Roman de Troie, t. I, v. 6658-6954, p. 352-371/Liet von Troye, v. 3973-4108, p. $46-47$. 
relatent l'arrivée de Pileüs et d'Acamus venus de Thrace ${ }^{38}$, si bien que Pretemesus et Steropeus («Crepeüs» dans $A I$ ), nommés immédiatement après dans l'énumération, arrivent de Thrace et non plus de Pannonie :

$$
\begin{aligned}
& \text { De Trace une terre pleniere } \\
& \text { dont la jant est hardie et fiere } \\
& \text { et adure et combatanz } \\
& \text { plus que nule autre jant vivant } \\
& \text { car es forez et es monteignes } \\
& \text { dont il i a plus que des pleignes } \\
& \text { voit an folez et satyriaus } \\
& \text { bestes sauvaiges et oisiaus } \\
& \text { de .m. menieres ou de plus : } \\
& \text { de la fu rois Protemissus } \\
& \text { et Crepeüs uns suens cosins. } \\
& \text { (AI, f. } 42 \mathrm{a})
\end{aligned}
$$

Il est remarquable que le texte du Liet von Troye présente le même raccourci et une forme semblable pour le nom de Steropeus :

$$
\begin{aligned}
& \text { Da was ouch von Traze, } \\
& \text { von schonem gelazze, } \\
& \text { der kunic Pretemisus } \\
& \text { und sin nefe Crepeus }{ }^{39} .
\end{aligned}
$$$$
\text { (v. 4013-4016) }
$$

Il est vrai que le manuscrit $A l$ omet en outre, dans le dénombrement, les vers du Roman de Troie décrivant l'arrivée de Pistropleus et du Sagittaire ${ }^{40}$ tandis que l'adaptateur allemand, tout en passant sous silence le Sagittaire, mentionne son maître ${ }^{41}$.

Au-delà des leçons particulières à $A l$ signalées par Marc-René Jung, l'on peut relever d'autres passages qui offrent des concordances entre les textes de $A 1$ et du Liet von Troye ${ }^{42}$. En premier lieu, le dénombre-

38. Le Roman de Troie, t. I, v. 6733-6748, p. 356-357.

39. Liet von Troye, p. 46 ( « Il y avait aussi, originaires de Thrace et de belle apparence, le roi Pretemesus et son neveu Steropeus $»)$.

40. Le Roman de Troie, t. I, v. 6893-6906, p. 367-368 / A1, f. 43a.

41. Liet von Troye, v. 4073-4079, p. 47.

42. Pour la suite de l'analyse, nous nous fondons, en ce qui concerne les manuscrits autres que $\boldsymbol{A l}$, sur les variantes qu'indique $\mathrm{L}$. Constans. Naturellement, l'éditeur ne pouvait, dans son immense entreprise, consigner toutes les 
ment des navires grecs ${ }^{43}$ qui précède le dénombrement des alliés de Priam fournit des indices supplémentaires autour d'Ajax, de Podalire et de Cuneüs.

Dans Le Roman de Troie, deux Ajax sont mentionnés au cours du dénombrement : Ajax fils de Télamon et Ajax fils d'Oïlée. Le texte précise au sujet du second :

$$
\begin{aligned}
& \text { Trente e set en ot li vassaus } \\
& \text { Oileïus Aïaus } \\
& \text { de Logres, sa terre demeine : } \\
& \text { n'i aveit celi ne fust pleine } \\
& \text { d'omes, d'armes e de vitaille, } \\
& \text { toz volenteïs de bataille. } \\
& \text { (éd. Constans, v. 5633-5638) }
\end{aligned}
$$

En cet endroit du texte, $A I$ comme le Liet von Troye se contentent de nommer le personnage en même temps que Capus :

Seisante set an ot Capus et Ayax Oyleüs.

$$
(A 1, \text { f. 35b) }
$$

$$
\begin{aligned}
& \text {...sehs und firzic Caspus } \\
& \text { mit dem andern Aiace } 44 .
\end{aligned}
$$

(v. 3344-3345)

$A I$ n'est pas le seul manuscrit en cela ${ }^{45}$. En revanche, il semble être le seul à évoquer de nouveau Ajax quelques dizaines de vers plus loin dans le dénombrement, entre l'évocation de Télépolème et celle d'Eurypylus ${ }^{46}$ :

Ayax de Logres i vint, qui tot a conte an i ot vint.

$$
(A 1,35 \mathrm{c})
$$

variantes : il donne entièrement les variantes de sept manuscrits en intégrant partiellement celles des autres (voir M.-R. Jung, op. cit., p. 17). Il faudrait donc, en toute rigueur, effectuer des recherches sur tous les manuscrits que $L$. Constans n'a pu prendre en compte.

43. Le Roman de Troie, t. I, v. 5583-5702, p. 292-301.

44. Liet von Troye, p. 39 (« Capus [en apporta] quarante-six avec l'autre Ajax 》).

45. Selon les variantes notées par $\mathrm{L}$. Constans, les manuscrits $D, M I, J, P, E$, $H, B 2, B 4$ et $S 3$ présentent aussi la forme capus, $A$ comporte caphus et e taphus (Le Roman de Troie, t. I, variantes du vers 5633, p. 296).

46. L'ajout s'insère entre les vers 5664 et 5665 de L. Constans, qui ne signale ici aucune variante (Le Roman de Troie, t. I, p. 298). 
C'est sans doute l'explication du troisième Ajax qui apparaît dans l'adaptation allemande à l'endroit correspondant du texte :

Der dritte Ayax gebeten wart, daz er in die hervart furte zwenzic schif fol 47 .

(v. 3377-3379)

On aura noté que le nombre de navires apportés par ce troisième Ajax est le même dans les textes de $A l$ et du Liet von Troye.

Par ailleurs, l'auteur du Liet von Troye mentionne dans le dénombrement deux Podalire, dont le second apparaît entre Euryale et Polibétès :

Polidarius der hete

mit gutem gerete,

als iz was vor bedacht,

funf und zwenzic dar brach[t] ${ }^{48}$.

(v. 3393-3396)

Le texte ainsi que l'apparat critique donnés par Léopold Constans ne nous apprennent rien sur ce personnage absent du Roman de Troie. En revanche, $A I$ possède un ajout à l'endroit correspondant du texte de Benoît 49 :

\section{Rois Polidarius an rot} .$x x v$. que meillors n'i ot.

$$
(A 1, \text { f. 35c) }
$$

Cette fois encore, non seulement $A l$ nous livre un éclaircissement sur l'origine du personnage présent dans le Liet von Troye, mais en outre le nombre de navires concorde dans les deux textes.

Quelques vers plus loin dans le même dénombrement, après la mention de Philoctète, le texte du Roman de Troie évoque Cuneüs :

47. Liet von Troye, p. 39 (« Le troisième Ajax fut prié de se joindre à l'expédition avec vingt navires remplis $")$.

48. Liet von Troye, p. 39-40 ( Podalire avait apporté vingt-cinq navires et du bon matériel, comme cela avait été prévu »). Le premier Podalire apparaît au vers 3363 .

49. Il s'agit d'un ajout entre les vers 5680 et 5681 du texte édité par L. Constans, qui signale par ailleurs un ajout de $A 2$ : "Dunt sire estoit rois Adrastus / Cui fille avoit dans Tydeus » (Le Roman de Troie, t. I, p. 299). 


\section{Uns reis de Cipe Cuneüs \\ onze en $\mathrm{i}$ ot e neient plus. \\ (éd. Constans, v. 5685-5686)}

Le Liet von Troye ne fait pas mention de ce personnage, qui d'après l'apparat critique de Léopold Constans est présent dans tous les manuscrits de Benoît. Le manuscrit $A l$ le remplace cependant par Hélénus :

$$
\begin{aligned}
& \text { Uns forz rois de Chispre Helenus } \\
& \text { an } \mathrm{i} \text { ot .x. et noiant plus. }
\end{aligned}
$$

$$
(A 1, \text { f. 35c) }
$$

C'est bien le personnage que l'on retrouve dans le passage correspondant du Liet von Troye, avec le même nombre de navires :

Von Kypre quamen auch do zehene mit Hele[n]o so.

(v. 3399-3400)

En outre, à la suite d'Hélénus, Herbort évoque Patrocle, alors que le texte de Léopold Constans mentionne Prothoüs : sur ce point aussi, $A 1$ concorde avec le texte allemand (il est vrai qu'il n'est pas le seul en cela ${ }^{51}$ ). De même, l'omission par Herbort d'Agapenor de Capadie, le personnage suivant dans Le Roman de Troie, se retrouve dans $A 1$ (comme dans trois autres manuscrits du Roman de Troie ${ }^{52}$ ). On pourra mettre en valeur l'ensemble des concordances regroupées dans cet extrait du dénombrement par la mise en regard des passages correspondants, dans l'édition de Léopold Constans, dans le manuscrit $A l$ et dans le texte allemand :

50. Liet von Troye, p. 40 (« De Chypre vinrent aussi à ce moment-là dix navires menés par Hélénus »).

51. Liet von Troye, v. 3401, p. 40 / Le Roman de Troie, t. I, v. 5689, p. 300 / A1, f. $35 \mathrm{c}$. D'après les variantes indiquées par $\mathrm{L}$. Constans, les manuscrits $A, K, M$ et $M 3$ comportent aussi le nom de Patrocle.

52. L'omission correspond aux vers 5691-5692 du texte édité par L. Constans : " Agapenor de Capadie / En rot cinquante en la navie » (Le Roman de Troie, t. I, p. 300)/ $A 1$, f. 35 c. Les autres manuscrits concernés sont $D, B I$ et M2. 
Ayax de Logres i vint,

Euripilus d'Orcomenie, uns reis de mout grant seignorie, en ot cinquante bien guarnies,

bien chargiees et bien emplies.

D'Elide, une terre sauvage,

en ot o sei onze al rivage

danz Antipus e Amphimaus :

n'en ert a dire tref ne maus.

Si com la letre me devise, seisante en i ot de Larise Polibetés e Leontins, qui esteient germains cosins.

Diomedés e Sthelenus e li beaus Eürialus $i$ aduistrent oitante barges de la cité, de l'onor d'Arges.

De la terre de Melibee, que donc n'ert guaires habitee, en $\mathrm{i}$ ot set Philotetès, qui mout esteit fel e engrès. Uns reis de Cipe Cuneüs

onze en $i$ ot e neient plus.

Cinquante en $i$ ot de Manese -

n'en $i$ aveit nule remese Prothoïlus, quin esteit sire, riches, poissanz de grant empire.

Agapenor de Capadie en rot cinquante en la navie [...].

(éd. Constans, v. 5665-5692 / A1, f. 35c / Liet von Troye, v. 3377-3402) qui tot a conte an $i$ ot vint.

Der dritte Ayax gebeten wart,

daz er in die hervart furte zwenzic schif fol. Euripilus, zu gereche wol,

Euripilus d'Orcomenie, uns rois de moult grant seignorie, an i ot .xv. bien garnies, Evor, als er zu der herfart geswur.

bien chargiees et bien amplies.

De Libe, une terre sauvaige, an i orent. $\mathrm{xx}$. au rivaige rois Xamtipus et Amphimas :

n'an ert a dire trez ne mas.

Si com la letre me devise,

.xl. an $i$ ot de Larise

Polibetés et Liochin, qui estoient germein cousin. Dyomedés et Stelenus et li biaus rois Eurialus i aduistrent .1 . barges de la terre et de l'enor d'Arges.

Rois Polidarius an rot .xxv. que meillors n'i ot. De la terre de Melibee, qui lors n'iert guieres abitee, an i ot .xx. Politetés, qui assez fu fal et angrés. Helenus an i ot .x. et noiant plus.

Von Lize brachte Cantipus drizehene und Anfimacus.

Lyochin mit Polipite brachten firzic uf den se mit geselleschaft gewisse. Der herre von Arysse, Dyomedes und Stelenus und der schone Euriolus furten firzic schiffe starke mit also maniger barke.

Polidarius der hete mit gutem gerete, als iz was vor bedacht, funf und zwenzic dar brach[t].

Auch quamen mit Politete sibene von Melibete.

Von Kypre quamen auch do zehene mit Hele[n]o. Uns forz rois de Chispre Meise -

ne n'an i a nule remeise -

Patroclus, qui an estoit sire, Patroclus dar gahte :

forz et poissanz de grant funfzic sch[if] er brachte ${ }^{53}$. ampire.

53. Liet von Troye, p. 39-40 (« Le troisième Ajax fut prié de se joindre à l'expédition avec vingt navires remplis. Eurypylus, bien préparé à la vengeance, vint avec cinquante navires lorsqu'il s'engagea pour l'expédition. D'Élide, Antipus et Amphimacus en apportèrent treize. Léontin et Polibétès en menèrent quarante sur la mer, ainsi que des hommes fiables. Le seigneur d'Argos, Dio- 
En somme, il semble que seul le manuscrit $A 1$ puisse expliquer trois des divergences relevées entre Le Roman de Troie et le Liet von Troye dans le dénombrement des navires grecs : la présence de deux Podalire et de trois Ajax ainsi que l'absence de Cuneüs. Les deux autres divergences, la substitution de Patrocle à Prothoüs et l'omission d'Agapenor de Capadie, peuvent également s'expliquer - mais non exclusivement il est vrai - par $A 1$.

En second lieu, à la suite du dénombrement des navires grecs, le dénombrement des alliés de Priam livre un indice supplémentaire. En effet, le texte allemand mentionne dans le cadre de ce dénombrement un certain "Funas de Philistea ${ }^{54}$ ». Ce personnage n'apparaît pas dans le texte édité par Léopold Constans, qui indique en cet endroit : "De Boëce i vint Asimas 55 ». Les variantes consignées dans l'édition n'indiquent rien qui concorde avec le texte de Herbort. En revanche, on lit dans le manuscrit $A I$ :

De Philistee i vint Phimas,

Fortins li prouz et Sanias :

conte estoient tuit por roi

mais ainz ne fierent porte foi.

$(A 1$, f. $42 \mathrm{~b})$

En dernier lieu, plus avant dans le texte, l'énumération des chefs grecs et troyens engagés dans la deuxième bataille ${ }^{56}$ offre trois autres concordances autour de Glaucon et Sarpédon, de Cadorz de Liz et d'Ascalophus.

mède et Sthénélus ainsi que le bel Euryale menèrent quarante navires solides et autant d'embarcations. Podalire avait apporté vingt-cinq navires et du bon matériel, comme cela avait été prévu. Arrivèrent également, avec Philoctète, sept navires de Melibée. De Chypre vinrent aussi à ce moment-là dix navires menés par Hélénus. Patrocle se hâta d'arriver; il apportait cinquante navires $\gg)$.

54. Liet von Troye, v. 4023, p. 46.

55. Le Roman de Troie, t. I, v. 6781 , p. 360 . Les variantes indiquées en note par $L$. Constans, qui donne ici le texte de $A$, sont : $B I$ boete, $B$ boice $; L$ bote i revint ; ( $B 1$ asimas, cf. v. $6782 H$ asimas, $M I$ asymas), $B P R$ afimas, $M 2 M$ afunas, $F$ aufumas, $J$ ansimas, $A 2 C E H$ anf., $G N$ amph., $M I$ anph., $L$ anfoinas.

56. Le Roman de Troie, t. I, v. 7641-8022, p. 408-434 / Liet von Troye, v. 4629-4790, p. 53-55. 
Tout d'abord, comme le note Léopold Constans, le manuscrit $A 1$ présente dans l'énumération des chefs troyens une leçon particulière sur douze vers ${ }^{57}$. Alors que les autres manuscrits mentionnent deux personnages du nom de Glaucon, le roi de Lycie et son fils Glaucon Fierejostice ${ }^{58}, A 1$ nomme ici Glaucon et Sarpédon, qu'il présente comme des frères, en accord avec un passage antérieur du texte ${ }^{59}$. Sur ce point aussi, le Liet von Troye s'accorde avec $A I$ :

Hector a pris deus suens amis qui si cousin erent proichien : andui erent frere jermein, li ainznez avoit non Glaucon, l'autre apeloit an Sarpedon; andui ierent roi queroné de Lice, dom il erent né. Cist feront andui vasselaige
Der kune Ector nam san under den fursten ware, we(i)lcher mit siner schare vor faren mochte, daz der da zu tochte. Do riet der kunic selbe, daz in der werlde nieman fugete baz den der kunic Glaucon

57. Ces vers correspondent aux vers 7693-7702 dans le texte édité par $\mathbf{L}$. Constans :

Hector a pris dous suens amis.

Li uns fu fiz al rei de Lice ;

Glaucon ot non Fierejostice :

cist fu mout beaus, cist fut mout sages ;

en cestui ot mout vasselages.

Parent li ert assez prochain,

ensi come fiz de s'antain.

Mil chevaliers esliz e buens,

que des son pere, que des suens,

a pris Glaucon a fereors, armez es chevaux milsoudors [...].

58. Glaucon Fierejostice n'apparaît que dans ce passage, d'après la table analytique des noms propres procurée par L. Constans (Le Roman de Troie, t. V, p. 54).

59. Il s'agit des vers 6685-6686 dans le texte édité par $L$. Constans : « De Lice i vint li vieux Glaucon / e sis fiz o lui Sarpedon ».

L'éditeur signale comme variantes pour $f i z: E k$ frere; $P$ freres (Le Roman de Troie, t. I, p. 354).

$A I$ comporte au f. 41d : «De lice i vint li rois glaucon / et ses freres danz sarpedon $»$.

Voici le passage correspondant dans le Liet von Troye : « der kunic von syze glaucon / und sin bruder sarpedon » (« Le roi de Lycie, Glaucon, et son frère Sarpedon... ", Liet von Troye, v. 3989-3990, p. 46). 
car molt estoient prou et saige. .M. chevaliers a pris de suens, chascuns d'ax esleüz et buens. $(A 1$, f. $47 \mathrm{~d}-48 \mathrm{a})$ und sin bruder Sarpedon.

Sie waren beide von Lizelant.

Sie scharten ir folc alda zu hant 60 .

(v. 4650-4660)

En outre, l'évocation des chefs troyens se clôt provisoirement sur l'énumération d'une partie des trente fils bâtards de Priam, laquelle est complétée dans un second temps au terme d'un intermède consacré à Hector ${ }^{61}$. Au début du second volet de l'énumération, l'auteur du Roman de Troie indique que douze ou treize bâtards - selon les manuscrits - ont déjà été nommés et qu'il en reste donc soit dix-huit soit dix-sept à mentionner. En réalité, seuls dix noms ont été donnés dans le premier volet de l'énumération, et dans le second, quel que soit le chiffre annoncé, dix-huit autres sont évoqués : au total, ce sont vingt-huit bâtards et non trente qui sont énumérés. Or l'adaptateur allemand fait remarquer, dans le passage correspondant du Liet von Troye, que trois des trente bâtards restent anonymes ${ }^{62}$, et en toute logique il nomme lui-même dix bâtards dans le premier volet de l'énumération puis dix-sept dans le second volet. La comparaison entre les dix-huit noms du modèle français et les dix-sept du texte allemand, dans le second volet de l'énumération, montre que c'est Cadorz de Liz, le seizième bâtard nommé par Benoît ${ }^{63}$, qui disparaît dans l'adaptation. $A 1$ une fois de plus nous éclaire sur cette omission puisqu'en lieu et place du vers « Sezimes fu Cadorz de Liz », le manuscrit précise au sujet du précédent bâtard de la liste : "Chevaliers ert boens et esliz ${ }^{64}$ ". C'est ainsi que la disparition du seizième bâtard, Cadorz de Liz, dans le texte de Herbort, peut s'expliquer par son absence dans le manuscrit $A I$.

60. Liet von Troye, p. 54 (« Le téméraire Hector se demanda bientôt qui, parmi les princes, pourrait partir en tête avec son armée, afin de choisir celui qui en fût capable. Le roi lui-même émit un conseil en disant que personne au monde ne conviendrait mieux que le roi Glaucon et son frère Sarpedon. Ils venaient tous deux du pays de Lycie. Ils rassemblèrent alors de suite leurs troupes »).

61. Le Roman de Troie, t. I, v. 8097-8134, p. 438-444 / Liet von Troye, v. $4821-4844$, p. 56.

62. Liet von Troye, v. 4811-4818, p. 56.

63. Le Roman de Troie, t. I, v. 8125 , p. 441.

64. $A 1,50 \mathrm{c}$. D'après les notes de L. Constans, seul le manuscrit $E$ omet aussi le personnage de Cadorz (Le Roman de Troie, t. V, p. 9). 
L'énumération des chefs grecs ${ }^{65}$ qui succède à celle des chefs troyens offre une concordance supplémentaire entre $A 1$ et le Liet von Troye. AI est en effet le seul manuscrit, d'après les variantes relevées par Léopold Constans, à présenter Machaon non comme le fils de Labius mais comme celui d'Ascalophus :

...Machaon, li fiz Labius

e li reis Polidarius...

(éd. Constans, v. 8263-8264) ...li fill le roi Asqualophi, cest Maquaon, et Pelidri...

$(A 1$, f. 51c)

Le texte du Liet von Troye s'accorde avec celui de $A 1$ :

...ein vater und sin son :

daz was der kunic Machaon

und sin vater Ascalosius,

mit in der kunic Polidius ${ }^{66}$.

(v. 4909-4912)

Pour finir, nous évoquerons le récit que fait Ulysse de ses aventures à la demande du roi Idoménée, car ce passage essentiel des « retours » comporte une particularité intéressante dans l'adaptation allemande : il se présente sous forme de discours direct, alors que Le Roman de Troie ne possède que quelques passages relatés sur ce mode ${ }^{67}$. Dans un article récent ${ }^{68}$, Michael Mecklenburg note que sur ce point, Herbort ne s'inscrit pas dans la veine de Darès et de Dictys comme le fait Benoît : curieusement, l'adaptateur allemand adopte le même procédé qu'Homère dans l'Odyssée, pourtant restée inconnue jusqu'au milieu du XIV ${ }^{\mathrm{e}}$ siècle. Michael Mecklenburg signale par ailleurs une particula-

65. Le Roman de Troie, t. I, v. 8165-8328, p. 444-455/ Liet von Troye, v. $4855-4935$, p. $56-57$.

66. Liet von Troye, p. 57 (« [Se joignirent également aux troupes] un père et son fils : c'était le roi Machaon et son père Ascalophus, accompagnés du roi Podalire $\gg)$.

67. Liet von Troye, v. 17544-17749, p. 200-203 / Le Roman de Troie, t. IV, v. 28591-28936, p. 295-314. Les passages au discours direct, dans l'édition de L. Constans, correspondent aux vers 28627-28643, 28679-28700 et 28923-28936.

68. M. Mecklenburg, « So horet wie ulixes sprach. Die Erzählung des Ulysses in Herborts von Fritzlar Liet von Troye », dans Erzählungen in Erzählungen. Phänomene der Narration in Mittelalter und Früher Neuzeit, H. Haferland / M. Mecklenburg ed., München, 1996 [Forschungen zur Geschichte der älteren deutschen Literatur, 19], p. 41-57. 
rité du manuscrit $A l$ en mentionnant que le manuscrit comporte du discours direct dans une partie du récit ${ }^{69}$. Le critique rejette toutefois la possibilité que $A I$ ait été la source de Herbort en raison de la date du manuscrit, et il revient pour finir à l'hypothèse du manuscrit $A 2$, en faisant référence aux recherches de Hermann Menhardt ${ }^{70}$. La consultation du manuscrit $A I$ révèle qu'en réalité, celui-ci présente du discours direct dès le début du récit d'Ulysse ${ }^{71}$ : c'est là une concordance supplémentaire avec l'adaptation allemande. Pour revenir en outre à l'argument chronologique évoqué par Michael Mecklenburg, il est vrai que $A 1$, écrit en 1237 selon une indication située après l'explicit ${ }^{72}$, ne peut être lui-même le manuscrit dont disposait Herbort. En revanche, il peut être un proche parent du manuscrit-source utilisé par l'adaptateur. Au reste, la comparaison entre le Liet von Troye et un manuscrit français postérieur ne pose pas de problème d'un point de vue méthodologique, car, selon Marc-René Jung, « il est évident que la diffraction en deux familles des manuscrits du Roman de Troie a dû se produire encore au XII ${ }^{\mathrm{e}}$ siècle ${ }^{73}$ ").

On voit toute l'importance que revêt l'établissement d'une base philologique rigoureuse pour l'in terprétation des divergences entre une adaptation allemande et son modèle. Avant d'affirmer, par exemple, que Herbort recourt au texte de Virgile pour corriger Le Roman de Troie, il convient de connaître la source utilisée par l'adaptateur. La question est particulièrement complexe dans le cas du Liet von Troye, non seulement de par le nombre de manuscrits qui transmettent $L e$ Roman de Troie, mais aussi en raison de la manière d'adapter propre à Herbort : la comparaison entre les deux textes s'avère souvent difficile tant l'auteur allemand abrège sa source romane. Néanmoins, les concordances présentées ci-dessus nous portent à croire que nous

69. Cette partie débute plus précisément au vers 28679 (M. Mecklenburg, art. cit., note 4, p. 42). D'après les variantes indiquées par L. Constans, $A l$ est le seul en cela. Seuls les manuscrits $M, H$ et $K$ semblent avoir, outre les passages au discours direct mentionnés à la note 67 , des formes verbales ou pronominales à la première personne (« costiasmes / costeiai », " prismes / pris ", « ne nous / ne me ") au début du passage (Le Roman de Troie, t. IV, v. 28606-28608, p. 296).

70. M. Mecklenburg, art. cit., note 4, p. 42.

71. Soit à partir du f. $179 \mathrm{~b}$.

72. F. 189 d.

73. Nous nous permettons ici de citer une lettre de M. Marc-René Jung, auquel nous exprimons notre vive reconnaissance pour son aide. 
possédons dans le manuscrit $A I$ du Roman de Troie une version proche du manuscrit-source du Liet von Troye. L'hypothèse mérite désormais d'être étudiée pour tout le texte ${ }^{74}$.

Marie-Sophie MAsse.

\section{L'ÉMERAUDE MINÉE Chrétien de Troyes ET LE LAPIDAIRE}

Le cinéma nous a habitués à être attentifs quand la caméra glisse comme par hasard sur quelque chose, un objet ou un geste. Le spectateur sait par expérience que le moment viendra où l'accessoire s'avérera essentiel.

Chrétien de Troyes aime le détail. Il peut même en donner plus que n'en demande le lecteur, quand, dans une description par exemple, il cumule les aspects précieux d'un objet ou quand il donne, dans une action, des explications même là où le lecteur, qu'il a habitué aux événements merveilleux et aux coups de théâtre, n'attend pas de commentaire.

Cette insistance peut sembler être un élément secondaire, de pure décoration, visant à enjoliver la riche façade d'une société aristocratique de quelques joyaux de plus. Elle peut aussi ajouter aux effets comiques de la narration quand Chrétien s'amuse à expliquer dans le détail le rayonnement du merveilleux, un excès qui provoque le sourire : lorsque Érec s'agenouille au pied de la Croix dans laquelle sont incrustées des escarboucles, l'auteur ne se contente pas, comme il le fait ailleurs, de louer l'extraordinaire éclat de ces pierres précieuses qui illuminent comme des soleils ${ }^{1}$, mais il va jusqu'à préciser que chaque

74. Telle est la tâche que nous avons entreprise.

1. Cf. la beauté de Fenice qui dans le roman Cligés «Rent el palés si grant clarté / Com feissent .IIII. escharbocle. " (v. 2704-2705, éd. C. MélaO. Collet, Paris, 1994). Cf. la description du lit de la merveille dans Perceval "A chascun des quepouz del lit / Ot une escharboucle fermé, / Qui randoit tres si grant clarté, / Cun quatre cierge bien espris » (v. 7622-7625, éd. C. Méla, Paris, 1990). 